\title{
Rostral Hypothalamic Differentiation With Minimal Exogenous Signals in Human Naive Induced Pluripotent Stem Cells
}

\section{Hajime Ozaki}

Department of Endocrinology and Diabetes, Nagoya University Graduate School of Medicine Hidetaka Suga ( $\sim$ sugahide@med.nagoya-u.ac.jp )

Department of Endocrinology and Diabetes, Nagoya University Graduate School of Medicine

\section{Mayu Sakakibara}

Department of Endocrinology and Diabetes, Nagoya University Graduate School of Medicine

\section{Mika Soen}

Department of Endocrinology and Diabetes, Nagoya University Graduate School of Medicine

\section{Natsuki Miyake}

Department of Obstetrics and Gynecology, Nagoya University Graduate School of Medicine

\section{Tsutomu Miwata}

Department of Endocrinology and Diabetes, Nagoya University Graduate School of Medicine

\section{Shiori Taga}

Regenerative \& Cellular Medicine Kobe Center, Sumitomo Dainippon Pharma Co., Ltd.

\section{Takashi Nagai}

Department of Obstetrics and Gynecology, Nagoya University Graduate School of Medicine

\section{Mayuko Kano}

Department of Endocrinology and Diabetes, Nagoya University Graduate School of Medicine Kazuki Mitsumoto

Department of Endocrinology and Diabetes, Nagoya University Graduate School of Medicine Takashi Miyata

Department of Endocrinology and Diabetes, Nagoya University Graduate School of Medicine Tomoko Kobayashi

Department of Endocrinology and Diabetes, Nagoya University Graduate School of Medicine

\section{Mariko Sugiyama}

Department of Endocrinology and Diabetes, Nagoya University Graduate School of Medicine

\section{Takeshi Onoue}

Department of Endocrinology and Diabetes, Nagoya University Graduate School of Medicine Hiroshi Takagi

Department of Endocrinology and Diabetes, Nagoya University Graduate School of Medicine

\section{Daisuke Hagiwara}


Department of Endocrinology and Diabetes, Nagoya University Graduate School of Medicine

\section{Shintaro Iwama}

Department of Endocrinology and Diabetes, Nagoya University Graduate School of Medicine

\section{Ryoichi Banno}

Department of Endocrinology and Diabetes, Nagoya University Graduate School of Medicine

\section{Genzo Iguchi}

Division of Diabetes and Endocrinology, Kobe University Hospital

\section{Yutaka Takahashi}

Division of Diabetes and Endocrinology, Department of Internal Medicine, Kobe University Graduate School of Medicine

\section{Keiko Muguruma}

RIKEN Center for Developmental Biology (CDB)

\section{Haruhisa Inoue}

Center for iPS Cell Research and Application (CiRA), Kyoto University

\section{Hiroshi Arima}

Department of Endocrinology and Diabetes, Nagoya University Graduate School of Medicine

\section{Research Article}

Keywords: neurohypophyseal, diabetes, degenerative, vasopressin, pathology

Posted Date: January 6th, 2022

DOI: https://doi.org/10.21203/rs.3.rs-1202298/v1

License: (c) (1) This work is licensed under a Creative Commons Attribution 4.0 International License. Read Full License 


\section{Abstract}

Familial neurohypophyseal diabetes insipidus (FNDI) is a degenerative disease of vasopressin (AVP) neurons. Studies in mouse in vivo models indicate that accumulation of mutant AVP prehormone is associated with FNDI pathology. However, studying human FNDI pathology in vivo is technically challenging. Therefore, an in vitro human model needs to be developed. When exogenous signals are minimized in the early phase of differentiation in vitro, mouse embryonic stem cells (ESCs) differentiate into AVP neurons, whereas human ESCs/induced pluripotent stem cells (iPSCs) die. Human ES/iPSCs are generally more similar to mouse epiblast stem cells compared to mouse ESCs, which are termed as primed and naive, respectively. In this study, we converted human FNDI-specific iPSCs from primed to naive cells, and found improved cell survival under minimal exogenous signals and differentiation into rostral hypothalamic organoids. Overall, this method provides a simple and straightforward differentiation direction, which may improve the efficiency of hypothalamic differentiation.

\section{Introduction}

The hypothalamus plays an essential role in maintaining physiological homeostasis. One of these is the generation and secretion of arginine vasopressin (AVP), which controls water balance. Familial neurohypophyseal diabetes insipidus (FNDI) is characterized by a progressive decrease in AVP secretion caused by the degeneration of neurons in the hypothalamus. Mutations in the AVP gene locus have been identified in patients with FNDI, and the majority of these mutations are located in the region encoding the AVP carrier protein, neurophysin II (NPII) ${ }^{1}$. Therefore, we generated FNDI mice with a Cys98stop (previously called Cys67stop) mutation ${ }^{2}$, which occurs in patients with $\mathrm{FNDI}^{3}$, for pathological analysis. Our previous studies using Cys98stop-knock-in FNDI mouse models ${ }^{4-13}$, indicated that endoplasmic reticulum (ER) stress caused by the accumulation of mutant proteins in the ER could be associated with FNDI pathology. However, further studies, including the use of human models, are needed to investigate the overall pathological mechanisms of FNDI. FNDI-related studies in humans are restricted by technical limitations because hypothalamic biopsies cannot be performed in living humans and the loss of AVP neurons in human patients with FNDI has been reported only in autopsy studies ${ }^{14-16}$. Therefore, AVP neurons derived from human FNDI-specific induced pluripotent stem cells (iPSCs) are a promising human model for pathological analysis and drug development.

Pluripotent stem cells can differentiate into various cells or tissues in three-dimensional cultures termed "serum-free culture of embryoid body-like aggregates with quick re-aggregation" (SFEBq) ${ }^{17-27}$. Based on the differentiation of hypothalamic neurons from mouse embryonic stem cells (mESCs) by SFEBq ${ }^{28}$, we improved the differentiation efficiency of mouse iPSCs ${ }^{29}$. In these methods, the differentiation of pluripotent stem cells into neural tissue is achieved by regulating the positional information added to the medium. The hypothalamus in the embryo originates from the most rostral part of the neural plate (Fig. S1A). To differentiate the hypothalamus from mouse ES/iPSCs, it is important to remove exogenous signals strictly in the early stages of differentiation, and to control the positional information so that it 
represents the most rostral side ${ }^{28,29}$. The medium with minimized exogenous signals is called a growth factor-free chemically defined medium ( $(\mathrm{fCDM})^{28}$. With gfCDM, mouse ES/iPSCs differentiated into the rostral hypothalamus, including AVP neurons.

However, this theoretical concept in mouse ES/iPSCs has not yet been realized in the differentiation of human ES cells (hESCs). Under conditions with minimal exogenous signals using gfCDM, hESCs cannot form aggregates and die within a few days. The addition of knock out serum replacement (KSR) allows hESCs to form aggregates, but results in their differentiation into telencephalic progenitor cells. This means that the positional information contained in KSR shifts the differentiating position to caudal and dorsal. Thus, to achieve hypothalamic differentiation, it is necessary to use KSR-containing medium to guide the cells toward the telencephalon in the first step, and then force them to change their position to the hypothalamus by adding rostralizing and ventralizing signals in the second step ${ }^{30}$ (Fig. S1B). We speculate that this two-step differentiation strategy causes less efficient differentiation to the hypothalamus. Therefore, it is necessary to improve the method for hESC/hiPSC differentiation into the hypothalamus. In this study, we focused on the difference in the starting points between hES/iPSCs and $\mathrm{mES} / \mathrm{iPSCs}$.

Mouse epiblast stem cells (mEpiSCs) derived from post-implantation embryos ${ }^{31,32}$ have characteristics different from those of conventional mESCs derived from pre-implantation embryos. Nichols et al. advocated the concept of 'naive' and 'primed', which correspond to mESCs and mEpiSCs, respectively ${ }^{33}$. Human ESCs are recognized to be in a primed state, similar to mEpiSCs. In fact, hESCs and mEpiSCs share the same colony morphology and vulnerability to isolation. Therefore, in this study, we examined the hypothesis that if hESCs/iPSCs were converted to a naive state, they could be differentiated in the same way as $\mathrm{mESCs}$.

\section{Results}

\section{Primed FNDI-specific hiPSCs fail to aggregate and differentiate poorly using conventional methods}

The differentiation of hypothalamic neurons from mESCs and hESCs was achieved by SFEBq ${ }^{28,30}$, but there were differences between the methods. With minimal exogenous signals, mESCs differentiated into hypothalamic neurons whereas hESCs failed to form aggregates. Therefore, following the hESC method, primed FNDI hiPSCs (FDI-02) were dissociated into single cells and suspended in gfCDM containing $5 \%$ KSR. Under these conditions, they aggregated quickly. When the concentration of KSR was maintained at $5 \%$, the aggregates grew without collapsing. However, as previously reported, maintaining the concentration of KSR at $5 \%$ without other exogenous signals caused the hESCs to differentiate into FOXG1-positive telencephalic progenitors ${ }^{30}$. Addition of BMP4 and SAG caused hESCs to differentiate into hypothalamic precursors, but at a lower efficiency than that in the method using mESCs with minimal exogenous signals 28,30 . To minimize the effects of exogenous signals, we reduced their 
concentrations and shortened their incubation period (Fig. S2A). When the concentration of KSR at day 0 was reduced to $2.5 \%$ or KSR-free gfCDM was used after day 3 , the aggregates collapsed or formed cysts (Fig. S2B).

Our previous study using miPSCs showed that stopping miPSC colonies to clumps of approximately 1020 cells, rather than isolating them to a single cell for SFEBq, can facilitate miPSC aggregation ${ }^{29}$. Accordingly, SFEBq using clumps was performed to prevent collapse and cyst formation. When the KSR concentration at day 0 was $2.5 \%$ and KSR-free gfCDM was used after day 3 , the clumps quickly aggregated and grew without collapsing. However, even under these conditions, primed FDI-02 differentiated into FOXG1-positive telencephalic progenitors (Fig. S2B). When a lower concentration of KSR was examined on day 0 , the aggregates collapsed or formed cysts. Although SFEBq using clumps could ease the effects of reduced KSR concentration to some extent, it was difficult for primed FDI-02 to differentiate into hypothalamic neurons with minimal exogenous signals.

\section{Primed-to-naive conversion improves the differentiation of FDI-02 into hypothalamic precursors}

To minimize the exogenous signals needed for FDI-02 differentiation, we assessed a method that enables human pluripotent stem cells to be treated in the same manner as mouse pluripotent stem cells.

Compared with conventional mESCs, human pluripotent stem cells show some distinctive features, including differences in developmental identity. In particular, mEpiSC derivation ${ }^{31,32}$ shows alternative pluripotency, which is associated with primitive streak-stage late epiblasts ${ }^{34}$. The terms 'naive' and 'primed,' which correspond to mESCs and mEpiSCs, respectively, refer to the early and late stages of epiblast ontogeny ${ }^{33}$. Human pluripotent stem cells share defining features with primed mEpiSCs rather than with naive mESCs. Takashima et al. and Guo et al. reported methods for converting cells from the

primed state to the naive state ${ }^{35-37}$. We hypothesized that these methods could help hiPSCs differentiate into hypothalamic neurons by minimizing exogenous signals.

Primed FDI-02 cells were cultured under feeder-free conditions using mTeSR1 ${ }^{\mathrm{TM}}$ culture medium. Thereafter, the medium was replaced with $\mathrm{RS}^{\mathrm{T}} \mathrm{T}^{\mathrm{TM}}$. The colony morphology changed from flat to tightly packed and domed, and TFCP2L1, a naive marker ${ }^{38}$, was expressed (Fig. 1A-D), confirming conversion from the primed to naive state.

SFEBq was performed with naive FDI-02 single cells and primed FDI-02 clumps. The use of a medium containing neither KSR nor Y-27632, a Rho-associated kinase (ROCK) inhibitor, from an early stage was compared with a medium containing both throughout the culture (Fig. 2A). Even when the initial concentration of KSR was reduced to $0.7 \%$, the single cells and clumps quickly aggregated under all conditions. Most primed aggregates formed cysts or collapsed over time, whereas naive aggregates 
maintained a healthy state. The naive aggregates showed significantly fewer changes on day 30 (Fig. 2B, C).

Aggregates were evaluated by immunohistochemistry. The expression of BRN2, an AVP precursor marker ${ }^{39,40}$, was significantly increased in naive aggregates in the shortened period condition with KSR and Y-27632 (Fig. 2D, E). This is consistent with increased hypothalamic differentiation with minimal exogenous signals.

In summary, conversion to the naive state enabled minimization of exogenous signals in SFEBq with hiPSCs. Furthermore, the minimization of exogenous signals improved the efficiency of differentiation into hypothalamic-like cells.

\section{Hypothalamic organoids derived from FDI-02 expressed mutant NPII}

Based on these results, the early stage of the differentiation protocol was optimized. We have previously reported that the addition of FGF8b to late-stage culture improves the efficiency of AVP neuron differentiation ${ }^{29}$. Therefore, we adopted this method and established an overall protocol (Fig. 3A).

SFEBq using the naive human iPSC cell line, 201B7, was performed according to the established protocol. Dorsal hypothalamic progenitor (PAX6+, NKX2.1-) and AVP precursor (OTP+, BRN2+) cells were sequentially identified (Fig. 3B, C). On day 150, we observed cells co-expressing AVP, NPII, and copeptin, which are components of pro-AVP, and thus achieved differentiation into AVP neurons (Fig. 3D). Using the same method, naive FDI-02 cells differentiated into AVP neurons. Simultaneously, we confirmed the expression of other hypothalamic markers such as CRF, NPY, AgRP, TRH, and Orexin (Fig. 3E-H), which demonstrated successful differentiation to AVP neurons as well as hypothalamic organoids. On day 150, we confirmed the expression of mutant NPII, which was not expressed in 201B7-derived AVP neurons (Fig. 4).

\section{Discussion}

In this study, we investigated a novel perspective in the differentiation of AVP neurons, regarding whether cells were in a naive or primed state before differentiation, rather than considering the early or later stages of the differentiation protocol ${ }^{28-30}$. We found two advantages. First, the conversion of cells from primed to naive solved problems such as decreased survival, collapse of aggregates, and cyst formation by minimizing exogenous signals in the SFEBq method for the differentiation of human pluripotent stem cells into AVP neurons. Second, the strict removal of exogenous signals by conversion to the naive type increased the rate of cells positive for BRN2, which is an essential transcription factor for AVP differentiation ${ }^{39-43}$. These findings indicate an improved efficiency of AVP neuron differentiation.

The differentiation potential of pluripotent stem cells varies among cell lines ${ }^{44}$. When differentiating the rostral part of the central nervous system such as the hypothalamus, the use of naive cells could be one way to solve this problem. It is difficult to differentiate the primed hESCs/hiPSCs into rostral 
hypothalamic neurons because that they cannot survive in culture conditions with minimal exogenous signals. Historically, it is known that dissociation of primed hESCs causes apoptosis due to the Rhohigh/Rac-low state ${ }^{45}$. Thus, treatment with ROCK inhibitors is very effective for the dissociation process of primed hESCs ${ }^{46}$. Using a ROCK inhibitor, primed hESCs/hiPSCs can be differentiated into non-rostral nervous systems such as the telencephalon ${ }^{17,18}$, cerebellum ${ }^{22}$, and spinal cord ${ }^{24}$. However, for inducing the rostral part of the central nervous system, such as AVP neurons, additional conditions are required to induce the rostral edge of the neural plate by using minimal exogenous signals as the positional information during differentiation ${ }^{28,29}$. In the primed hESC/hiPSC cultures, use of the ROCK inhibitor alone cannot maintain their aggregation in the state of minimal exogenous signals. Nutrients such as KSR must be added to some extent for their survival, resulting in their differentiation into FOXG1-positive cerebral tissues. Additional BMP4 and SHH signals are necessary to primed hESCs/hiPSCs for

repositioning from the cerebrum to the hypothalamus ${ }^{30}$. This involves a two-step process: once the cells are posteriorized, they are repositioned anteriorly. This is thought to be the reason for the previously low efficiency of hypothalamic differentiation. By converting hiPSCs from the primed-type to the naive type, addition of ROCK inhibitor and KSR can be reduced to the minimum, resulting in a simpler straightforward differentiation direction, which may improve the efficiency of differentiation into the rostral hypothalamus.

Analyses of our FNDI model mice, which possess the Cys98stop mutation ${ }^{2,3}$, revealed that mutant NPII was accumulated in the ER-associated compartment (ERAC) in AVP neurons ${ }^{4,5}$. In this study, we clearly demonstrated mutant NPII expression in FNDI-specific hiPSC-derived AVP neurons. Accumulation of mutant proteins in the ER is implicated in the pathophysiology of many diseases, including $\mathrm{FNDI}^{1,6-}$ 13,47,48; therefore, FNDI-specific hiPSC-derived AVP neurons are a promising human model of ER stress and are a valuable resource for drug development. We plan to analyze characteristic structures, such as the ERAC, which was confirmed in mice by electron microscopy, in FNDI-specific hiPSC-derived AVP neurons. We would also like to undertake a functional investigation for pathological analysis and drug development in future studies.

In this study, conversion from the prime to naive state was performed based on the reports by Takashima et al. and Guo et al. ${ }^{35-37}$. However, there are several other reports concerning conversion to the naive type ${ }^{49-57}$ and a novel concept termed 'formative type' has also been advocated between the naive and primed types ${ }^{58}$. Although we achieved some success by inducing conversion to the naive type, further studies are needed to determine the optimal state of the cells when initiating differentiation from hiPSCs. Based on this study, we will continue to improve the differentiation method, analyze the expression pattern of mutant proteins in detail, and research drug reactivity.

\section{Methods}

These experiments were approved by the ethics committee of Nagoya University and performed in accordance with relevant guidelines and regulations. Informed consent was obtained from all subjects. 


\section{Generation of FNDI hiPSCs and a control cell line}

We generated iPSCs from a patient with FNDI with a Cys98Stop mutation ${ }^{3}$ based on ethical approval from Nagoya University Committee (2013-0228-2). To establish patient-derived iPSCs, peripheral blood monocular cells of the patient were reprogrammed using episomal vectors (expressing OCT3/4, SOX2,

KLF4, L-MYC, LIN28, EBNA1, and p53shRNA) as described previously ${ }^{59,60}$. The iPSCs showed embryonic stem cell-like morphology and normal karyotype (Fig. S3A). These cells expressed the undifferentiated markers (Fig. S3B, C) and could differentiate into the three germ layers in vitro (Fig. S3D), indicating that these cells were pluripotent (deposited in RIKEN BRC as HPS1011 and HPS1904). We named them as FDI-02. As a control, we used the 201B7 cell line [Research Resource Identifier (RRID): CVCL_A324].

\section{Thawing and maintenance of primed hiPSCs}

We prepared mouse embryonic fibroblasts (MEFs) inactivated by mitomycin $C$ treatment on $0.1 \%$ gelatincoated dishes $\left(1.2 \times 10^{6} \mathrm{cells} / 10 \mathrm{~cm}\right.$ dish). Primed hiPSCs frozen in liquid nitrogen were thawed as quickly as possible in warmed maintenance medium, comprising DMEM/F12 (D6421; Sigma, St. Louis, Missouri, USA) supplemented with 20\% (v/v) KSR (lot No. 1517496; Invitrogen, Waltham, Massachusetts, USA), 0.1 mM non-essential amino acids (11140-050; Gibco, Waltham, MA, USA), 2 mM L-glutamine (25030-081; Gibco), 5 ng/mL recombinant human basic FGF (068-04544; Wako, Osaka, Japan), and 0.1 mM 2-mercaptoethanol (131-14572; Wako. Cells suspended in maintenance medium were distributed on MEF-coated dishes and maintained in a $\mathrm{CO}_{2}$ incubator under $2 \% \mathrm{CO}_{2}$ at $37^{\circ} \mathrm{C}$. The medium was changed daily.

For passaging, primed hiPSC colonies were harvested by incubation in $0.25 \%(\mathrm{w} / \mathrm{v})$ trypsin and 0.1 $\mathrm{mg} / \mathrm{mL}$ collagenase IV in PBS containing $20 \%(\mathrm{v} / \mathrm{v}) \mathrm{KSR}$ and $1 \mathrm{mM} \mathrm{CaCl}_{2}$ for $6-8 \mathrm{~min}$ at $37^{\circ} \mathrm{C}$. The harvested clumps were broken into smaller pieces by gentle pipetting. The passages were performed at a split ratio of 1:4-6.

\section{Reset of hiPSCs from the primed state to naive state and maintenance incubation}

hiPSCs harvested during passaging, were suspended in $\mathrm{mTeSR} 1^{\mathrm{TM}}$ medium (ST-85850; STEMCELL Technologies, Vancouver, Canada), distributed on a Matrigel-coated dish (354277; Corning, Corning, New York, USA), and incubated under $5 \% \mathrm{CO}_{2}$ at $37^{\circ} \mathrm{C}$. After $24-36 \mathrm{~h}$, the mTeSR 1 medium was replaced with RSeT $^{\text {TM }}$ feeder-free medium (ST-05975; STEMCELL Technologies) and cells were incubated under hypoxic conditions $\left(5 \% \mathrm{O}_{2}, 5 \% \mathrm{CO}_{2}\right)$ at $37^{\circ} \mathrm{C}$. The medium was changed every second day. Reset to the naive state was confirmed by changes in colony morphology and immunohistochemistry.

For passaging, naive hiPSCs were harvested using TrypLE Express (12605-010; Thermo Fisher, Waltham, Massachusetts, USA). Cells were counted and $1.1 \times 10^{6}$ cells were distributed on a $10 \mathrm{~cm}$ Matrigel-coated dish. The medium was changed after $24-36 \mathrm{~h}$ and thereafter, every second day. This passaging procedure was performed every 4-6 days. 


\section{Thawing and maintenance of primed hiPSCs}

Hypothalamic differentiation was performed using the SFEBq culture. Primed hiPSCs were harvested and dissociated into single cells using TrypLE Express (12605-010; Invitrogen) containing $0.05 \mathrm{mg} / \mathrm{mL}$ DNase I (11284932001; Roche, Basel, Switzerland) and 10 mM Y-27632 (034-24024; Wako). Cells were then suspended in gfCDM differentiation medium [1:1 Iscove's-modified Dulbecco's medium (IMDM), GlutaMAX ${ }^{\mathrm{TM}}$ supplement (31980-030; Gibco)/Ham's F-12 nutrient mix, GlutaMAX' ${ }^{\mathrm{TM}}$ supplement (31765035; Gibco), 250 mg/mL BSA (A3156; Sigma), 1× chemically defined lipid concentrate (11905-031; Gibco), and $438 \mu \mathrm{M}$ 1-thioglycerol (M6145; Sigma)] and distributed in low-cell-adhesion 96-well plates with V-bottomed conical wells (MS-9096V; Sumitomo Bakelite, Tokyo, Japan) at 5,000 cells in 100

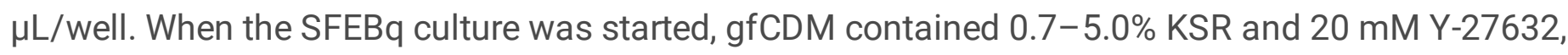
which were diluted in a step-wise manner by replacing one-half of the medium with new medium without KSR or Y-27632. In another method, harvested primed hiPSCs were broken by gentle pipetting. Eighty percent of the broken clumps from one confluent $10 \mathrm{~cm}$ dish were suspended in gfCDM and distributed into one 96-well plate. Naive hiPSCs were harvested using TrypLE Express and dissociated into single cells without mechanical disruption. These were then suspended in gfCDM (5,000 cells in $100 \mu \mathrm{L} /$ well) and distributed in 96 -well plates. All SFEBq cultures were incubated in $5 \% \mathrm{CO}_{2}$ at $37^{\circ} \mathrm{C}$. The concentrations of KSR and Y-27632 in the medium used for the medium change were adjusted for each experiment.

We designated the day when the culture was started as day 0 . On day $3,100 \mu \mathrm{L}$ gfCDM was added to each well. From day 6 , half of the medium was changed every 3 days. After culturing in a 96 -well plate for 30 days, the aggregates were transferred to a $10 \mathrm{~cm}$ dish for suspension culture. From day 60,100 ng/mL FGF8b (423-F8; R\&D Systems, Minneapolis, MN, USA) and $5 \mu \mathrm{g} / \mathrm{mL}$ heparin (NIPRO, Osaka, Japan) were added to the differentiation medium. From day 90 , the medium was completely replaced with DMEM/F12 supplemented with glucose, N2, and B27 [DFNB medium: DMEM/F12 (D8900; Sigma) supplemented with $3.85 \mathrm{~g} / \mathrm{L}$ glucose (07-0680-5; Sigma), $1.2 \mathrm{~g} / \mathrm{L}$ sodium hydrogen carbonate (28-1850-5; Sigma), penicillin/streptomycin (50 U/mL and $50 \mu \mathrm{g} / \mathrm{mL}$, respectively) (15140-122; Gibco), N2 (17502001; Gibco), B27 (125870-01; Gibco), and $10 \mathrm{ng} / \mathrm{mL}$ CNTF (257-NT; R\&D Systems)] with $100 \mathrm{ng} / \mathrm{mL}$ FGF8b and $5 \mu \mathrm{g} / \mathrm{mL}$ heparin.

\section{Immunohistochemistry}

Aggregates were fixed with 4\% PFA for 10-15 min and embedded in OCT compound (4583; Sakura Finetek, Tokyo, Japan). Ten micron thick sections were cut using a cryostat, mounted on slides, and fixed in $4 \%$ PFA for 10 min.

Sections were permeabilized using $0.3 \%$ Triton X-100/PBS and washed with PBS. Subsequently, they were incubated in $2 \%(\mathrm{w} / \mathrm{v})$ skimmed milk/PBS for $1 \mathrm{~h}$ at RT and then with primary antibodies diluted in $2 \%$ skimmed milk/PBS, overnight at $4^{\circ} \mathrm{C}$. The next day, they were washed with $0.05 \%$ Tween $20 / \mathrm{PBS}$ and reacted with 4,6-diamidino-2-phenylindole (DAPI; D523; Dojindo, Kumamoto, Japan) and secondary 
antibodies diluted in $2 \%$ skimmed milk/PBS for $2 \mathrm{~h}$ at RT. Subsequently, they were washed with $0.05 \%$ Tween 20/PBS and mounted in SlowFade ${ }^{\mathrm{TM}}$ Diamond (S36972; Thermo Fisher Scientific).

Primary antibodies were used against the following molecules (with dilutions): AVP (T5048; guinea pig; 1:2000; Peninsula; RRID:AB_2313978), BRN2 (sc-6029; goat; 1:500; Santa Cruz; RRID:AB_2167385), Copeptin (BORIS Y; rabbit; 1:1000; Woomera Therapeutics), E-cad (M108; rat; 1:50; TAKARA), FOXG1 (M227; rabbit; 1:1000; TAKARA; RRID:AB_2827749), mutant NPII [AFT965002-B(2B); rabbit; 1:1000; custom], NANOG (4903; rabbit; 1:500; Cell signaling; RRID:AB_10559205), NKX2.1 (16108; mouse; 1:100; PROGEN; RRID:AB_1543129), NPII (MABN845; mouse; 1:1000; Millipore; RRID:AB_2819363), OCT3/4 (611202; mouse; 1:100; BD biosciences; RRID:AB_398736), OTP (MS1535GS; guinea pig; 1:1000; Takara), PAX6 (ab195045; rabbit; 1:350; abcam; RRID:AB_2750924), SMA (M0851; mouse; 1:500; DAKO; RRID:AB_2223500), SOX17 (81778; rabbit; 1:3000; Cell Signaling; RRID:AB_2650582), SSEA-4 (MAB4304; mouse; 1:2000; Millipore; RRID:AB_177629), TFCP2L1 (AF5726; goat; 1:200; R\&D Systems; RRID:AB_2202564), TRA1-60 (MAB4360; mouse; 1:200; Millipore; RRID:AB_2119183), and TUJ1 (MMS435P; mouse; 1:500; Covance; RRID:AB_2313773).

\section{Statistics and reproducibility}

We have described the exact $n$ values for each experiment in the main text and figure legends. IBM SPSS Statistics (IBM, Armonk, New York, USA) was used for the statistical analyses. Two-group comparisons were performed using the two-tailed unpaired $t$ test. Significance was set at $\mathrm{P}<0.05$.

\section{Declarations}

\section{Data Availability}

The datasets generated during and/or analyzed during this study are available from the corresponding author upon reasonable request.

\section{Acknowledgments}

We are grateful to Ayaka Nishiyama for establishment of human FNDI-specific iPSCs. We thank Akiko Tsuzuki for technical assistance, and all members of the Arima laboratory for valuable discussions. We would like to thank Editage (www.editage.com) for English language editing.

This work was supported by grants from the Program for Intractable Disease Research Utilizing DiseaseSpecific iPS Cells from the Japan Science and Technology (JST) agency and the Japan Agency for Medical Research and Development (AMED) (K.M, JP15bm0404009, JP16bm0609002), the Project for Technological Development (to H.S.) of the Research Center Network for Realization of Regenerative Medicine (RCNRRM), funded by AMED (JP17bm0404018, JP20bm0404036), the Acceleration Program for Intractable Diseases Research Utilizing Disease-Specific iPS Cells (to H.S.) of RCNRRM funded by AMED (JP19bm0804011), Grants-in-Aid for Scientific Research (to H.S.) from the Ministry of Education, Culture, Sports, Science, and Technology of Japan (MEXT; JP17K09878, JP20K08859), Nagoya 
University Hospital Funding for Clinical Research (71004136; to H.S.), Fusion Oriented Research for disruptive Science and Technology (FOREST) by Japan Science and Technology Agency (JST), the Hori Sciences and Arts Foundation, the Toyoaki Scholarship Foundation, the Daiko Foundation, the Nitto Foundation, and the Suzuken Memorial Foundation.

Author information:

Affiliations

Department of Endocrinology and Diabetes, Nagoya University Graduate School of Medicine H. Ozaki, H. Suga, M. Sakakibara, M. Soen, T. Miwata, S. Taga, M. Kano, K. Mitsumoto, T. Miyata, T. Kobayashi, M. Sugiyama, T. Onoue, H. Takagi, D. Hagiwara, S. Iwama, R. Banno \& H. Arima

Department of Obstetrics and Gynecology, Nagoya University Graduate School of Medicine

N. Miyake \& T. Nagai

Regenerative \& Cellular Medicine Kobe Center, Sumitomo Dainippon Pharma Co., Ltd.

S. Taga

Division of Diabetes and Endocrinology, Kobe University Hospital

G. Iguchi

Medical Center for Student Health, Kobe University

G. Iguchi

Division of Diabetes and Endocrinology, Department of Internal Medicine, Kobe University Graduate School of Medicine

Y. Takahashi

Department of Diabetes and Endocrinology, Nara Medical University

Y. Takahashi

RIKEN Center for Developmental Biology (CDB)

K. Muguruma

Department of iPS Cell Applied Medicine, Graduate School of Medicine, Kansai Medical University

K. Muguruma 
Center for iPS Cell Research and Application (CiRA), Kyoto University

H. Inoue

iPSC-Based Drug Discovery and Development Team, RIKEN BioResource Research Center (BRC)

H. Inoue

Medical-risk Avoidance based on iPS Cells Team, RIKEN Center for Advanced Intelligence Project (AIP)

H. Inoue

\section{Contributions}

H. O. performed cell culture, immunohistochemistry, made all figures, and wrote the majority of the manuscript. H. S. conceived and designed the study and edited the manuscript. M. Sakakibara and M. Soen provided technical support. T. Miwata and K. M. discussed all experimental results. N. M., S. T., T. N. and M. K. provided useful advice on the SFEBq method. T. Miyata, H. T. and D. H. regularly advised on the results and figures. T. K., M. S., T. O., S. I., R. B. reviewed the manuscript. G.I. and Y, T. recruited FNDI patient. K. M. and H. I. generated FNDI-specific iPSCs. H. A. led and directed this study. All co-authors discussed and commented on the manuscript.

Corresponding author

Correspondence to Hidetaka Suga.

Ethics declarations:

Competing interests

The authors declare the following competing interests: Shiori Taga is an employee of Sumitomo Dainippon Pharma Co., Ltd. The other authors declare no competing interests.

\section{References}

1. Arima, H., Azuma, Y., Morishita, Y. \& Hagiwara, D. Central diabetes insipidus. Nagoya J Med Sci. 78, 349-357 (2016).

2. Hayashi, M. et al. Progressive polyuria without vasopressin neuron loss in a mouse model for familial neurohypophysial diabetes insipidus. Am J Physiol Regul Integr Comp Physiol. 296, R16411649 (2009).

3. Nagasaki, H. et al. Two novel mutations in the coding region for neurophysin-II associated with familial central diabetes insipidus. J Clin Endocrinol Metab. 80, 1352-1356 (1995).

4. Hagiwara, D. et al. Arginine vasopressin neuronal loss results from autophagy-associated cell death in a mouse model for familial neurohypophysial diabetes insipidus. Cell Death Dis. 5, e1148 (2014). 
5. Miyata, T. et al. Degradation of Mutant Protein Aggregates within the Endoplasmic Reticulum of Vasopressin Neurons. iScience 23, 101648 (2020).

6. Arima, H., Morishita, Y., Hagiwara, D., Hayashi, M. \& Oiso, Y. Endoplasmic reticulum stress in vasopressin neurons of familial diabetes insipidus model mice: Aggregate formation and mRNA poly(A) tail shortening. Exp Physiol. 99, 66-71 (2014).

7. Arima, H. \& Oiso, Y. Mechanisms underlying progressive polyuria in familial neurohypophysial diabetes insipidus. J Neuroendocrinol. 22, 754-757 (2010).

8. Azuma, Y. et al. Activating transcription factor $6 \mathrm{a}$ is required for the vasopressin neuron system to maintain water balance under dehydration in male mice. Endocrinology 155, 4905-4914 (2014).

9. Hagiwara, D., Grinevich, V. \& Arima, H. A novel mechanism of autophagy-associated cell death of vasopressin neurons in familial neurohypophysial diabetes insipidus. Cell and Tissue Res. 375, 259266 (2019).

10. Hiroi, M. et al. Activation of vasopressin neurons leads to phenotype progression in a mouse model for familial neurohypophysial diabetes insipidus. Am J Physiol Regul Integr Comp Physiol. 298, R486-493 (2010).

11. Kawaguchi, Y. et al. Endoplasmic reticulum chaperone BiP/GRP78 knockdown leads to autophagy and cell death of arginine vasopressin neurons in mice. Sci Rep. 10, 19730 (2020).

12. Morishita, Y. et al. Poly (A) tail length of neurohypophysial hormones is shortened under endoplasmic reticulum stress. Endocrinology 152, 48-46-4855 (2011).

13. Tochiya, M. et al. Chemical chaperone 4-phenylbutylate reduces mutant protein accumulation in the endoplasmic reticulum of arginine vasopressin neurons in a mouse model for familial neurohypophysial diabetes insipidus. Neurosci Lett. 682, 50-55 (2018).

14. Bergeron, C., Kovacs, K., Ezrin, C. \& Mizzen, C. Hereditary diabetes insipidus: an immunohistochemical study of the hypothalamus and pituitary gland. Acta Neuropathol. 81, 345348 (1991).

15. BRAVERMAN, L. E., MANCINI, J. P. \& MCGOLDRICK, D. M. HEREDITARY IDIOPATHIC DIABETES INSIPIDUS. A CASE REPORT WITH AUTOPSY FINDINGS. Ann Intern Med. 63, 503-508 (1965).

16. Nagai, I., Li, C. H., Hsieh, S. M., Kizaki, T. \& Urano, Y. Two cases of hereditary diabetes insipidus, with an autopsy finding in one. Acta Endocrinol. 105, 318-323 (1984).

17. Eiraku, M. et al. Self-Organized Formation of Polarized Cortical Tissues from ESCs and Its Active Manipulation by Extrinsic Signals. Cell Stem Cel/ 3, 519-532 (2008).

18. Kadoshima, T. et al. Self-organization of axial polarity, inside-out layer pattern, and species-specific progenitor dynamics in human ES cell-derived neocortex. Proc Natl Acad Sci US A. 110, 2028420289 (2013).

19. Kano, M. et al. Tanycyte-Like Cells Derived from Mouse Embryonic Stem Culture Show Hypothalamic Neural Stem/Progenitor Cell Functions. Endocrinology 160, 1701-1718 (2019). 
20. Kasai, T. et al. Hypothalamic Contribution to Pituitary Functions Is Recapitulated In Vitro Using 3DCultured Human iPS Cells. Cell Rep. 30, 18-24.e5 (2020).

21. Matsumoto, R. et al. Congenital pituitary hypoplasia model demonstrates hypothalamic OTX2 regulation of pituitary progenitor cells. J Clin Invest. 130, 641-654 (2019).

22. Muguruma, K., Nishiyama, A., Kawakami, H., Hashimoto, K. \& Sasai, Y. Self-Organization of Polarized Cerebellar Tissue in 3D Culture of Human Pluripotent Stem Cells. Cell Rep. 10, 537-550 (2015).

23. Ochiai, H. et al. BMP4 and FGF strongly induce differentiation of mouse ES cells into oral ectoderm. Stem Cell Res. 15, 290-298 (2015).

24. Ogura, T., Sakaguchi, H., Miyamoto, S. \& Takahashi, J. Three-dimensional induction of dorsal, intermediate and ventral spinal cord tissues from human pluripotent stem cells. Development 145 , dev162214 (2018).

25. Ozaki, H., Suga, H. \& Arima, H. Hypothalamic-pituitary organoid generation through the recapitulation of organogenesis. Dev Growth Differ. 63, (2021) 154-165.

26. Ozone, C. et al. Functional anterior pituitary generated in self-organizing culture of human embryonic stem cells. Nat Commun, 7, 10351 (2016).

27. Suga, H. et al. Self-formation of functional adenohypophysis in three-dimensional culture. Nature 480, 57-62 (2011).

28. Wataya, T. et al. Minimization of exogenous signals in ES cell culture induces rostral hypothalamic differentiation. Proc Natl Acad Sci U S A. 105, 11796-801 (2008).

29. Mitsumoto, K. et al. Improved methods for the differentiation of hypothalamic vasopressin neurons using mouse induced pluripotent stem cells. Stem Cell Res. 40, 101572 (2019).

30. Ogawa, K. et al. Vasopressin-secreting neurons derived from human embryonic stem cells through specific induction of dorsal hypothalamic progenitors. Sci Rep. 8, 3615 (2018).

31. Brons, I. G. M. et al. Derivation of pluripotent epiblast stem cells from mammalian embryos. Nature 448, 191-195 (2007).

32. Tesar, P. J. et al. New cell lines from mouse epiblast share defining features with human embryonic stem cells. Nature 448, 196-199 (2007).

33. Nichols, J. \& Smith, A. Naive and Primed Pluripotent States. Cell Stem Cell 4, 487-492 (2009).

34. Tsakiridis, A. et al. Distinct Wnt-driven primitive streak-like populations reflect in vivo lineage precursors. Development 141, 1209-1221 (2014).

35. Guo, G. et al. Naive Pluripotent Stem Cells Derived Directly from Isolated Cells of the Human Inner Cell Mass. Stem Cell Reports 6, 437-446 (2016).

36. Guo, G. et al. Epigenetic resetting of human pluripotency. [published correction appears in Development 145, dev166397 (2018)] Development 144, 2748-2763 (2017).

37. Takashima, Y. et al. Resetting transcription factor control circuitry toward ground-state pluripotency in human. Cell 158, 1254-1269 (2014). 
38. Martello, G., Bertone, P. \& Smith, A. Identification of the missing pluripotency mediator downstream of leukaemia inhibitory factor. EMBO J. 32, 2548-2560 (2013).

39. Alvarez-Bolado, G. Development of neuroendocrine neurons in the mammalian hypothalamus. Cell Tissue Res. 375, 23-39 (2019).

40. Nakai, S. et al. The POU domain transcription factor Brn-2 is required for the determination of specific neuronal lineages in the hypothalamus of the mouse. Genes Dev. 9, 3109-3121 (1995).

41. Xie, Y. \& Dorsky, R. I. Development of the hypothalamus: Conservation, modification and innovation. Development 144, 1588-1599 (2017).

42. Duplan, S. M., Boucher, F., Alexandrov, L. \& Michaud, J. L. Impact of Sim1 gene dosage on the development of the paraventricular and supraoptic nuclei of the hypothalamus. Eur J Neurosci. 30, 2239-2249 (2009).

43. Wang, W. \& Lufkin, T. The murine Otp homeobox gene plays an essential role in the specification of neuronal cell lineages in the developing hypothalamus. Dev Biol. 227, 179-185 (2000).

44. Ortmann, D. \& Vallier, L. Variability of human pluripotent stem cell lines. Curr Opin Genet Dev. 46, 179-185 (2017).

45. Ohgushi, M. et al. Molecular Pathway and Cell State Responsible for Dissociation-Induced Apoptosis in Human Pluripotent Stem Cells. Cell Stem Cell 7, 225-239 (2010).

46. Watanabe, K. et al. A ROCK inhibitor permits survival of dissociated human embryonic stem cells. Nat biotechnol. 25, 681-686 (2007).

47. Hotamisligil, G. S. Endoplasmic Reticulum Stress and the Inflammatory Basis of Metabolic Disease. Cell 140, 900-917 (2010).

48. Remondelli, P. \& Renna, M. The endoplasmic reticulum unfolded protein response in neurodegenerative disorders and its potential therapeutic significance. Front Mol Neurosci. 10, 187 (2017).

49. Chan, Y. S. et al. Induction of a human pluripotent state with distinct regulatory circuitry that resembles preimplantation epiblast. Cell Stem Cell 13, 663-675 (2013).

50 . Chen, H. et al. Reinforcement of STAT3 activity reprogrammes human embryonic stem cells to naivelike pluripotency. Nat Commun. 6, 7095 (2015).

51. Gafni, O. et al. Derivation of novel human ground state naive pluripotent stem cells. Nature 504, 282286 (2013).

52. Hanna, J. et al. Human embryonic stem cells with biological and epigenetic characteristics similar to those of mouse ESCs. Proc Natl Acad Sci U S A. 107, 9222-9227 (2010).

53. Qin, H. et al. YAP Induces Human Naive Pluripotency. Cell Rep. 14, 2301-2312 (2016).

54. Theunissen, T. W. et al. Systematic identification of culture conditions for induction and maintenance of naive human pluripotency. Cell Stem Cell 15, 471-487 (2014).

55. Wang, W. et al. Rapid and efficient reprogramming of somatic cells to induced pluripotent stem cells by retinoic acid receptor gamma and liver receptor homolog 1. Proc Natl Acad Sci U S A. 108, 18283- 
18288 (2011).

56. Ware, C. B. et al. Derivation of naïve human embryonic stem cells. Proc Natl Acad Sci U S A. 111, 4484-4489 (2014).

57. Zimmerlin, L. et al. Tankyrase inhibition promotes a stable human naïve pluripotent state with improved functionality. Development 143, 4368-4380 (2016).

58. Smith, A. Formative pluripotency: The executive phase in a developmental continuum. Development 144, 365-373 (2017).

59. Okita, K. et al. A more efficient method to generate integration-free human iPS cells. Nat Methods. 8 , 409-412 (2011).

60. Okita, K. et al. An efficient nonviral method to generate integration-free human-induced pluripotent stem cells from cord blood and peripheral blood cells. Stem Cells 31, 458-466 (2013).

\section{Figures}

\section{Figure 1}

Conversion of primed hiPSCs to naive pluripotency. (A) Primed hiPSC colonies had a flat morphology. Conversion from primed hiPSCs to naive pluripotency, changed the morphology of colonies to tightly packed and domed (B), and TFCP2L1 (green), a naive marker, was expressed (C, D). Scale bars, (A, B) 100 $\mu \mathrm{m},(\mathrm{C}, \mathrm{D}) 50 \mu \mathrm{m}$.

\section{Figure 2}

Naive iPSCs show improved aggregation on SFEBq and differentiation into hypothalamic-like cells. (A) Scheme of the culture protocol. Using a medium containing neither KSR nor Y-27632 from an early stage or a medium containing KSR and Y-27632 throughout, single naive hiPSCs (white arrows) were compared with primed hiPSC clumps (blue arrows). (B, C) On day 30, naive aggregates showed significantly fewer changes, such as cyst formation or collapse $(n=3)$. Mean $\pm S E M$. ${ }^{*} P<0.05 ; * * P<0.01$, two-tailed unpaired t-test. On day 30, primed aggregates scarcely expressed BRN2; however, BRN2 expression was observed by conversion to the naive state (D), and was significantly increased by shortening the period of KSR and Y-27632 treatment $(n=8)(E)$. Mean \pm SEM. ${ }^{*} P \mathrm{P}<0.01$, two-tailed unpaired $t$-test. OTP (green), BRN2 (red), FOXG1 (white). The increased expression of the AVP precursor marker, BRN2, indicated that minimization of exogenous signals improved the efficiency of differentiation into hypothalamic-like cells. For all relevant panels, nuclear counterstaining was performed with DAPI (blue). Scale bars, $100 \mu \mathrm{m}$. 


\section{Figure 3}

Naive hiPSCs differentiate into AVP neurons and hypothalamic organoid. (A) Scheme of the culture protocol. SFEBq using 201B7 cells recapitulated embryogenesis; on day 30, dorsal hypothalamic progenitor cells [PAX6+ (green), NKX2.1- (red)] (B), and on day 60, AVP precursor cells [OTP+ (green), BRN2+ (red)] (C), were sequentially identified. FOXG1 (white). (D) On day 150, differentiation into AVP neurons was achieved, with co-expression of pro-AVP components: AVP (green), NPII (red), and copeptin (white). Day 150 aggregates also expressed other hypothalamic markers such as CRF (E), NPY, AgRP (F), TRH (G), and Orexin $(H)$. It is demonstrated that these cells are not only AVP neurons but also hypothalamic organoids. For all relevant panels, nuclear counterstaining was performed with DAPI (blue). Scale bars, (B) $100 \mu \mathrm{m},(\mathrm{C}-\mathrm{H}) 50 \mu \mathrm{m}$.

\section{Figure 4}

FNDI-specific hiPSCs generate mutant NPII. Expression of normal NPII (red) was observed in SFEBq from FDI-02. Additionally, the expression of mutant NPII (mNPII) (green) was confirmed, which was not expressed in 201B7-derived AVP neurons. For all relevant panels, nuclear counterstaining was performed with DAPI (blue). Scale bars, $50 \mu \mathrm{m}$.

\section{Supplementary Files}

This is a list of supplementary files associated with this preprint. Click to download.

- FigureS1.tif

- FigureS2.tif

- FigureS3.tif 